\title{
On Spectral Representation of Varma Models with Change in Regime
}

\author{
Maddalena Cavicchioli
}

\author{
Dept. of Economics,University of Modena and Reggio Emilia, Italy \\ ${ }^{*}$ Corresponding Author: maddalena.cavicchioli@unimore.it
}

Copyright (C)2014 Horizon Research Publishing All rights reserved.

\begin{abstract}
We present various formulae in closed form for the spectral density of multivariate or univariate ARMA models subject to Markov switching, and describe some new properties of them. Many examples and numerical applications are proposed to illustrate the behaviour of the spectral density. This turns out to be useful in order to investigate various concepts of stationarity via spectral analysis.
\end{abstract}

Keywords Markov Switching VARMA, Spectral Density, Spectral Representation

JEL Classification: C32

\section{Markovian representation and spectral density}

Since the seminal papers by Hamilton (1989) (1990), multivariate ARMA models subject to Markov switching have been used actively in econometrics to model various time series. Stationarity, existence of moments and geometric ergodicity for such processes have been studied by several authors. See, for example, Francq and Zakoïan (2001) and Stelzer (2009). For estimation and consistency of these models we refer to Krishnamurthy and Ryden (1998) and Xie et al. (2008). A method to derive the spectral density matrix of such models was proposed by Pataracchia (2008) (2011). A formula in closed form for the spectral density matrix of these processes was given in Cavicchioli (2013), together with some new properties of it. Basic definitions and standard results on Matrix Analysis can be found, for example, in Horn and Johnson (1985) (1994). Let us consider a $d$-state Markov switching $K$-dimensional $\operatorname{ARMA}(p, q)$ model (in short, MS-VARMA $(p, q)$ ):

$$
\mathbf{x}_{t}=\sum_{i=1}^{p} a_{i}\left(\xi_{t}\right) \mathbf{x}_{t-i}+\boldsymbol{\epsilon}_{t}+\sum_{j=1}^{q} b_{j}\left(\xi_{t}\right) \boldsymbol{\epsilon}_{t-j}
$$

where $\mathbf{x}_{t}$ is a $K$-dimensional random vector with values in $\mathbb{R}^{K},\left(\xi_{t}\right)$ is an irreducible, aperiodic and ergodic Markov chain with finite space $\Xi=\{1,2, \ldots, d\}$, stationary transition probabilities denoted by $p_{i j}=\operatorname{pr}\left(\xi_{t}=j \mid \xi_{t-1}=i\right)$ and unconditional (or steady state) probabilities $\pi_{i}=\operatorname{pr}\left(\xi_{t}=i\right)$ for $i \in \Xi$. The $a_{i}\left(\xi_{t}\right)$ and $b_{j}\left(\xi_{t}\right)$ are $K \times K$ real random matrices. To allow for the possibility of change in variance, it is assumed that $\boldsymbol{\epsilon}_{t}=\boldsymbol{\epsilon}_{t}\left(\xi_{t}\right)=\sigma\left(\xi_{t}\right) \boldsymbol{\eta}_{t}$, where $\sigma\left(\xi_{t}\right)$ is a $K \times K$ real random matrix and $\left(\boldsymbol{\eta}_{t}\right)$ is a white noise with $E\left(\boldsymbol{\eta}_{t} \boldsymbol{\eta}_{t}^{\prime}\right)=\Omega$, the covariance matrix $\Omega$ being nonsingular. In addition, assume that $\left(\boldsymbol{\eta}_{t}\right)$ is independent of $\left(\xi_{t}\right)$. In order to investigate the stationarity properties of the process $\left(\mathbf{x}_{t}\right)$, Francq and Zakoïan (2001) proposed the following Markovian representation of (1):

$$
\mathbf{z}_{t}=\mathbf{\Phi}_{t} \mathbf{z}_{t-1}+\omega_{t} \quad \boldsymbol{\omega}_{t}=\boldsymbol{\Sigma}_{t} \boldsymbol{\eta}_{t} \quad \boldsymbol{\eta}_{t} \sim \operatorname{IID}(\mathbf{0}, \Omega)
$$

where:

$$
\mathbf{z}_{t}=\left(\begin{array}{llllllll}
\mathbf{x}_{t}^{\prime} & \mathbf{x}_{t-1}^{\prime} & \cdots & \mathbf{x}_{t-p+1}^{\prime} & \boldsymbol{\epsilon}_{t}^{\prime} & \boldsymbol{\epsilon}_{t-1}^{\prime} & \cdots & \boldsymbol{\epsilon}_{t-q+1}^{\prime}
\end{array}\right)^{\prime} \in \mathbb{R}^{K(p+q)}
$$




$$
\begin{aligned}
& \boldsymbol{\Sigma}_{t}=\left(\begin{array}{llllllll}
\sigma^{\prime}\left(\xi_{t}\right) & 0^{\prime} & \cdots & 0^{\prime} & \sigma^{\prime}\left(\xi_{t}\right) & 0^{\prime} & \cdots & 0^{\prime}
\end{array}\right)^{\prime} \in \mathcal{M}(K(p+q), K ; \mathbb{R}) \\
& \mathbf{A}_{t}=\left(\begin{array}{ccccc}
a_{1}\left(\xi_{t}\right) & a_{2}\left(\xi_{t}\right) & \cdots & a_{p-1}\left(\xi_{t}\right) & a_{p}\left(\xi_{t}\right) \\
I_{K} & 0 & \cdots & 0 & 0 \\
\vdots & \vdots & & \vdots & \vdots \\
0 & 0 & \cdots & I_{K} & 0
\end{array}\right) \in \mathcal{M}(K p ; \mathbb{R}) \\
& \mathbf{B}_{t}=\left(\begin{array}{cccc}
b_{1}\left(\xi_{t}\right) & b_{2}\left(\xi_{t}\right) & \cdots & b_{q}\left(\xi_{t}\right) \\
0 & 0 & \cdots & 0 \\
\vdots & \vdots & & \vdots \\
0 & 0 & \cdots & 0
\end{array}\right) \in \mathcal{M}(K p, K q ; \mathbb{R}) \\
& \mathbf{J}=\left(\begin{array}{ccccc}
0 & 0 & \cdots & 0 & 0 \\
I_{K} & 0 & \cdots & 0 & 0 \\
0 & I_{K} & \cdots & 0 & 0 \\
\vdots & \vdots & & \vdots & \vdots \\
0 & 0 & \cdots & I_{K} & 0
\end{array}\right) \in \mathcal{M}(K q ; \mathbb{R})
\end{aligned}
$$

and

$$
\mathbf{\Phi}_{t}=\left(\begin{array}{cc}
\mathbf{A}_{t} & \mathbf{B}_{t} \\
\mathbf{0} & \mathbf{J}
\end{array}\right) \in \mathcal{M}(K(p+q) ; \mathbb{R}) .
$$

Here $\mathcal{M}(m, n ; \mathbb{R})$ denotes the vector space formed by all $m \times n$ real matrices. In order to avoid degeneracies in $(2)$, we assume, without loss of generality, $p \geq 1$. In the case of a purely autoregressive model $(q=0)$, it is understood that $\mathbf{J}$ and $\mathbf{B}_{t}$ vanish, $\mathbf{z}_{t}=\left(\mathbf{x}_{t}^{\prime} \cdots \mathbf{x}_{t-p+1}^{\prime}\right)^{\prime}, \boldsymbol{\Sigma}_{t}=\left(\sigma^{\prime}\left(\xi_{t}\right) 0^{\prime} \cdots 0^{\prime}\right)^{\prime}$ and $\boldsymbol{\Phi}_{t}=\mathbf{A}_{t}$. Let $\Phi(i), A(i), B(i)$, and $\Sigma(i)$ be the matrices obtained by replacing $\xi_{t}$ by $i$ in $\boldsymbol{\Phi}_{t}, \mathbf{A}_{t}, \mathbf{B}_{t}$, and $\boldsymbol{\Sigma}_{t}$, respectively. Then the following matrices are defined:

$$
P=\left(\begin{array}{cccc}
p_{11}\{\Phi(1) \otimes \Phi(1)\} & p_{21}\{\Phi(1) \otimes \Phi(1)\} & \cdots & p_{d 1}\{\Phi(1) \otimes \Phi(1)\} \\
p_{12}\{\Phi(2) \otimes \Phi(2)\} & p_{22}\{\Phi(2) \otimes \Phi(2)\} & \cdots & p_{d 2}\{\Phi(2) \otimes \Phi(2)\} \\
\vdots & \vdots & & \vdots \\
p_{1 d}\{\Phi(d) \otimes \Phi(d)\} & p_{2 d}\{\Phi(d) \otimes \Phi(d)\} & \cdots & p_{d d}\{\Phi(d) \otimes \Phi(d)\}
\end{array}\right)
$$

which belongs to $\mathcal{M}\left(d K^{2}(p+q)^{2} ; \mathbb{R}\right)$ and

$$
S=\left(\begin{array}{c}
\pi_{1}\{\Sigma(1) \otimes \Sigma(1)\} \\
\pi_{2}\{\Sigma(2) \otimes \Sigma(2)\} \\
\vdots \\
\pi_{d}\{\Sigma(d) \otimes \Sigma(d)\}
\end{array}\right) \in \mathcal{M}\left(d K^{2}(p+q)^{2}, K^{2} ; \mathbb{R}\right)
$$

Let $P^{*}$ be the $[d K(p+q)] \times[d K(p+q)]$ matrix obtained by replacing $\Phi(i) \otimes \Phi(i)$ by $\Phi(i)$ in the definition of $P$. By Costa et al. (2005), Proposition 3.6, p.35, we see that $\rho(P)<1$ implies $\rho\left(P^{*}\right)<1$, where $\rho(\cdot)$ denotes the spectral radius. It can be easily checked that the converse is not true in general. See Remark 3.7, p.35, of the quoted book. In fact, for $p_{11}=p_{22}=0.5, \Phi(1)=a(1)=0.7$ and $\Phi(2)=a(2)=1.25$, we get $\rho(P)=1.02625$ and $\rho\left(P^{*}\right)=0.975$. The following theorem gives the necessary and sufficient conditions for the existence of second-order stationary Markov switching VARMA processes.

Theorem 1 (Francq and Zakoïan (2001)). Suppose that

$$
\rho(P)<1 \text {. }
$$

Then, for all $t \in \mathbb{Z}$, the series

$$
\mathbf{z}_{t}=\boldsymbol{\omega}_{t}+\sum_{k=1}^{+\infty} \boldsymbol{\Phi}_{t} \boldsymbol{\Phi}_{t-1} \cdots \boldsymbol{\Phi}_{t-k+1} \boldsymbol{\omega}_{t-k}
$$

converges in $L^{2}$ and the process $\left(\mathbf{x}_{t}\right)$, defined as the first component of $\left(\mathbf{z}_{t}\right)$, is the unique second-order stationary solution of (1). A necessary and sufficient condition for the existence of a second-order stationary solution of (1) is given by

$$
\sum_{k=0}^{+\infty}\left\|\Lambda P^{k} S \operatorname{vec} \Omega\right\|<+\infty
$$


where $\Lambda=\left(I_{K^{2}(p+q)^{2}} \cdots I_{K^{2}(p+q)^{2}}\right) \in \mathcal{M}\left(K^{2}(p+q)^{2}, d K^{2}(p+q)^{2} ; \mathbb{R}\right)$ and $\|\cdot\|$ denotes the matrix norm $\|M\|=$ $\sum_{i, j}\left|m_{i j}\right|$ for any matrix $M=\left(m_{i j}\right)$.

From now on, we assume $\rho(P)<1$, hence $\rho\left(P^{*}\right)<1$. Once second-order stationarity is ensured, it can be useful to compute the autocovariance $\Gamma_{\mathbf{x}}(k)$ of $\left(\mathbf{x}_{t}\right)$ for $k \in \mathbb{Z}$. For any $k \in \mathbb{Z}$, let us consider the matrix

$$
\mathcal{W}(k)=\left(\begin{array}{c}
\pi_{1} E\left[\mathbf{z}_{t} \mathbf{z}_{t-k}^{\prime} \mid \xi_{t}=1\right] \\
\pi_{2} E\left[\mathbf{z}_{t}^{\prime} \mathbf{z}_{t-k}^{\prime} \mid \xi_{t}=2\right] \\
\vdots \\
\pi_{d} E\left[\mathbf{z}_{t} \mathbf{z}_{t-k}^{\prime} \mid \xi_{t}=d\right]
\end{array}\right) \in \mathcal{M}(d K(p+q), K(p+q) ; \mathbb{R})
$$

By Francq and Zakoïan (2001), p.349, for all $k \geq 0$, we have

$$
\begin{aligned}
\Gamma_{\mathbf{x}}(k) & =\left(g^{\prime} \otimes f^{\prime}\right) \mathcal{W}(k) f \quad \mathcal{W}(k)=P^{* k} \mathcal{W}(0) \\
V & =\operatorname{vec} \mathcal{W}(0)=\left(I_{d K^{2}(p+q)^{2}}-P\right)^{-1} S \operatorname{vec} \Omega
\end{aligned}
$$

where $g=\left(\begin{array}{llll}1 & 1 & \cdots & 1\end{array}\right)^{\prime} \in \mathbb{R}^{d}$ and $f^{\prime}=\left(\begin{array}{llll}I_{K} & 0 & \cdots & 0\end{array}\right) \in \mathcal{M}(K, K(p+q) ; \mathbb{R})$. In particular, the variance-covariance matrix of $\left(\mathbf{x}_{t}\right)$ is given by $\operatorname{vec} \Gamma_{\mathbf{x}}(0)=\left(g^{\prime} \otimes f^{\prime} \otimes f^{\prime}\right) V$. For $k<0$, we have $\Gamma_{\mathbf{x}}(k)=\Gamma_{\mathbf{x}}^{\prime}(-k)$ as $C o v\left(\mathbf{x}_{t}, \mathbf{x}_{t-k}\right)=$ $\operatorname{Cov}\left(\mathbf{x}_{t-k}, \mathbf{x}_{t}\right)^{\prime}=\operatorname{Cov}\left(\mathbf{x}_{t}, \mathbf{x}_{t+k}\right)^{\prime}$ for any stationary process. As stated in Pataracchia (2011), p.13, we can compute the diagonal terms of the spectral density matrix $F_{\mathbf{x}}(\omega)$ of $\left(\mathbf{x}_{t}\right)$ by considering the summation

$$
F_{\mathbf{x}}(\omega)=\sum_{k=-\infty}^{+\infty}\left(g^{\prime} \otimes f^{\prime}\right) P^{*|k|} \mathcal{W}(0) f e^{-i \omega k}
$$

where the frequency $\omega$ belongs to $[-\pi, \pi]$. This series converges as $\rho\left(P^{*}\right)<1$. The following theorem gives a formula in closed form for the spectral density matrix of the process in (1).

Theorem 2 (Cavicchioli (2013)). Under the above notation, the spectral density matrix $F_{\mathbf{x}}(\omega)$ of the process $\left(\mathbf{x}_{t}\right)$ in (1) is given by

$$
F_{\mathbf{x}}(\omega)=\left(g^{\prime} \otimes f^{\prime}\right)\left(-I_{d K(p+q)}+2 \mathcal{R} e X(\omega)\right) \mathcal{W}(0) f
$$

where

$$
X(\omega)=\left(I_{d K(p+q)} e^{i \omega}-P^{*}\right)^{-1} e^{i \omega} \in \mathcal{M}(d K(p+q) ; \mathbb{C})
$$

and $\mathcal{R} e X(\omega)$ denotes the real part of $X(\omega)$. In the univariate case with $\Omega=1$, we get the even real-valued function

$$
f_{x}(\omega)=f^{\prime} \otimes\left[\left(g^{\prime} \otimes f^{\prime}\right)\left(-I_{d(p+q)}+2 \mathcal{R} e X(\omega)\right)\right]\left(I_{d(p+q)^{2}}-P\right)^{-1} S .
$$

If an observed time series $\mathbf{x}_{1}, \ldots, \mathbf{x}_{T}$ can be described by an $\operatorname{MS}-\operatorname{VARMA}(p, q) \operatorname{model}$, one good approach for estimating the population spectrum is to estimate the parameters of the model and substitute them into the expression of $F_{\mathbf{x}}(\omega)$.

\section{Explicit matrix computations}

(2.1) MS-AR(1). Let us consider a 2-state univariate AR(1) with Markov regime

$$
x_{t}=a\left(\xi_{t}\right) x_{t-1}+\epsilon_{t}
$$

where $\epsilon_{t} \sim \mathcal{N}\left(0, \sigma_{\epsilon}^{2}\right)$. By using Theorem 2, we explicitly compute now the spectral generating function $f_{x}(\omega)$ for the process in (4). Similar computations were given in Pataracchia (2008) but our formula is much simpler than the one obtained in the quoted paper. In this case, we have $K=1, d=2, p=1, q=0, \Omega=1, \Sigma=\sigma_{\epsilon}^{2}, \Phi(1)=a(1)$ and $\Phi(2)=a(2)$. The matrices $P$ and $S$ are given by

$$
P=\left(\begin{array}{ll}
p_{11}[a(1)]^{2} & p_{21}[a(1)]^{2} \\
p_{12}[a(2)]^{2} & p_{22}[a(2)]^{2}
\end{array}\right) \quad S=\sigma_{\epsilon}^{2}\left(\begin{array}{l}
\pi_{1} \\
\pi_{2}
\end{array}\right)
$$

where $p_{12}=1-p_{11}, p_{21}=1-p_{22}, \pi_{1}=\left(1-p_{22}\right)\left(2-p_{11}-p_{22}\right)^{-1}$ and $\pi_{2}=\left(1-p_{11}\right)\left(2-p_{11}-p_{22}\right)^{-1}$. The matrix

$$
\mathcal{W}(0)=\left(\begin{array}{l}
\pi_{1} E\left[x_{t}^{2} \mid \xi_{t}=1\right] \\
\pi_{2} E\left[x_{t}^{2} \mid \xi_{t}=2\right]
\end{array}\right)
$$


is easily computable. From $\mathcal{W}(0)=V=\left(I_{2}-P\right)^{-1} S$, we get

$$
\mathcal{W}(0)=\frac{\sigma_{\epsilon}^{2}}{\varphi_{P}(1)}\left(\begin{array}{l}
H \\
K
\end{array}\right)
$$

where

$$
\begin{aligned}
& H=\pi_{1}\left(1-p_{22}[a(2)]^{2}\right)+\pi_{2} p_{21}[a(1)]^{2} \\
& K=\pi_{1} p_{12}[a(2)]^{2}+\pi_{2}\left(1-p_{11}[a(1)]^{2}\right)
\end{aligned}
$$

Here $\varphi_{P}(\cdot)$ denotes the characteristic polynomial of $P$, hence

$$
\varphi_{P}(1)=\operatorname{det}\left(I_{2}-P\right)=1-\operatorname{tr}(P)+\operatorname{det}(P)
$$

Since

$$
P^{*}=\left(\begin{array}{ll}
p_{11} a(1) & p_{21} a(1) \\
p_{12} a(2) & p_{22} a(2)
\end{array}\right)
$$

we have

$$
Q(\omega)=\left(\begin{array}{ll}
1 & 1
\end{array}\right)\left(-I_{2}+2 \mathcal{R} e X(\omega)\right)=\left(\frac{Y(\omega)}{\left|\varphi_{P^{*}}\left(e^{i \omega}\right)\right|^{2}} \quad \frac{Z(\omega)}{\left|\varphi_{P^{*}}\left(e^{i \omega}\right)\right|^{2}}\right)
$$

where

$$
\begin{aligned}
Y(\omega) & =-\left|\varphi_{P^{*}}\left(e^{i \omega}\right)\right|^{2}+2 \mathcal{R} e\left[e^{-i \omega} \varphi_{P^{*}}\left(e^{i \omega}\right)\left(e^{-i \omega}+\left(p_{12}-p_{22}\right) a(2)\right)\right] \\
Z(\omega) & =-\left|\varphi_{P^{*}}\left(e^{i \omega}\right)\right|^{2}+2 \mathcal{R} e\left[e^{-i \omega} \varphi_{P^{*}}\left(e^{i \omega}\right)\left(e^{-i \omega}+\left(p_{21}-p_{11}\right) a(1)\right)\right] .
\end{aligned}
$$

Here we have

$$
\varphi_{P^{*}}\left(e^{i \omega}\right)=\operatorname{det}\left(I_{2} e^{i \omega}-P^{*}\right)=e^{2 i \omega}-\operatorname{tr}\left(P^{*}\right) e^{i \omega}+\operatorname{det}\left(P^{*}\right) .
$$

Then the spectral density function for the process in (4) has the following closed form

$$
f_{x}(\omega)=Q(\omega) \mathcal{W}(0)=\frac{\sigma_{\epsilon}^{2}}{\varphi_{P}(1)\left|\varphi_{P^{*}}\left(e^{i \omega}\right)\right|^{2}}\{H Y(\omega)+K Z(\omega)\}
$$

where $H$ and $K$ (resp. $Y(\omega)$ and $Z(\omega)$ ) are given by (5) (resp. (6)). It is an even real-valued function, so $\omega$ can be taken in $[0, \pi]$. Formula (7) is much simpler than the one obtained by Pataracchia (2008), Formula (12) in Section 3 and Appendix B. Formula (7) shows that the spectral density function of a 2-state MS-AR(1) presents itself as a sort of weighted average spectra of the underlying models. See also the pictures in the next section. For Model (4), local stationarity implies global stationarity. In fact, if $|a(i)|<1$ for $i=1,2$, then

$$
\rho(P) \leq \max \left\{p_{11}[a(1)]^{2}+p_{12}[a(2)]^{2}, p_{21}[a(1)]^{2}+p_{22}[a(2)]^{2}\right\}<1
$$

The converse is not true in general, i.e., we can have global stationarity without local stationarity within each regime. For example, for $p_{11}=0.2, p_{22}=0.4, a(1)=1.2$ and $a(2)=0.5$, we get $\rho(P) \sim 0.62\left(\right.$ and $\left.\rho\left(P^{*}\right) \sim 0.76\right)$. Finally, $\rho(P)<1$ is a necessary and sufficient condition for global stationarity of the process in (4). See Francq and Zakoïan (2001), Example 3, p.351.

(2.2) MS-ARMA $(1,1)$. Let us consider a 2-state univariate $\operatorname{ARMA}(1,1)$ with Markov switching

$$
x_{t}=a\left(\xi_{t}\right) x_{t-1}+\epsilon_{t}+b\left(\xi_{t}\right) \epsilon_{t-1}
$$

where $\epsilon_{t} \sim \mathcal{N}\left(0, \sigma_{\epsilon}^{2}\right)$. As before, we compute explicitly the spectral generating function $f_{x}(\omega)$ for the process $\left(x_{t}\right)$ in (8). In this case, we have $K=1, d=2, p=q=1, \Omega=1, \Sigma=\left(\begin{array}{ll}\sigma_{\epsilon} & 0\end{array}\right)^{\prime}$ and

$$
\Phi(1)=\left(\begin{array}{cc}
a(1) & b(1) \\
0 & 0
\end{array}\right) \quad \Phi(2)=\left(\begin{array}{cc}
a(2) & b(2) \\
0 & 0
\end{array}\right) .
$$

The matrices $P$ and $S$ are given by

$$
P=\left(\begin{array}{ll}
p_{11}\{\Phi(1) \otimes \Phi(1)\} & p_{21}\{\Phi(1) \otimes \Phi(1)\} \\
p_{12}\{\Phi(2) \otimes \Phi(2)\} & p_{22}\{\Phi(2) \otimes \Phi(2)\}
\end{array}\right)
$$

and

$$
S=\sigma_{\epsilon}^{2}\left(\begin{array}{llllllll}
\pi_{1} & 0 & 0 & 0 & \pi_{2} & 0 & 0 & 0
\end{array}\right)^{\prime}
$$


where $p_{i j}$ and $\pi_{i}, i, j=1,2$, are as in Subsection (2.1). The nonzero eigenvalues of the above $8 \times 8$ matrix $P$ coincide with those of the $2 \times 2$ matrix $P$ from Subsection (2.1). So these matrices have the same spectral radius. Furthermore, the previous discussion on stationarity of MS-AR(1) models maintains his validity also for MS-ARMA $(1,1)$. Now we can apply the formula in Theorem 2 where $f=\left(\begin{array}{ll}1 & 0\end{array}\right)^{\prime}, g=\left(\begin{array}{ll}1 & 1\end{array}\right)^{\prime}$ and

$$
P^{*}=\left(\begin{array}{ll}
p_{11} \Phi(1) & p_{21} \Phi(1) \\
p_{12} \Phi(2) & p_{22} \Phi(2)
\end{array}\right)
$$

Then we get

$$
\begin{aligned}
f_{x}(\omega)= & \sigma_{\epsilon}^{2} \frac{\left(1-p_{11}\right)\left(1-p_{22}\right)[a(1)]^{2}+\left(1-p_{22}\right)\left(1-p_{22}[a(2)]^{2}\right)}{\left(2-p_{11}-p_{22}\right) \varphi_{P}(1)} \\
& \times\left(-1+2 \mathcal{R} e\left\{\frac{1+\left(1-p_{11}\right) a(2) e^{i \omega}-p_{22} a(2) e^{i \omega}}{e^{4 i \omega} \varphi_{P^{*}}\left(e^{-i \omega}\right)}\right\}\right)
\end{aligned}
$$

where

$$
\varphi_{P}(1)=\operatorname{det}\left(I_{8}-P\right)=1-p_{11}[a(1)]^{2}-p_{22}[a(2)]^{2}-\left(1-p_{11}-p_{22}\right)[a(1)]^{2}[a(2)]^{2}
$$

and

$$
\varphi_{P^{*}}\left(e^{-i \omega}\right)=e^{-2 i \omega}\left[e^{-2 i \omega}-\left(p_{11} a(1)+p_{22} a(2)\right) e^{-i \omega}-\left(1-p_{11}-p_{22}\right) a(1) a(2)\right] .
$$

It is interesting to note that the coefficients $b\left(\xi_{t}\right)$ of the MA part in Model (8) do not appear in the expression of $f_{x}(\omega)$ given in (9). The knowledge of an explicit formula for the spectral density function of an MS-ARMA(1,1) model allows us to obtain the spectral representations for Markov Switching Generalized Autoregressive Conditional Heteroschedastic models (in short, MS-GARCH(1,1)) and for Markov Switching Stochastic Volatility models (in short, MS-SV). In fact, it is well-known that these last processes admit MS-ARMA $(1,1)$ reprersentations via linearization.

(2.3) MS-VARMA $(p, q)$. Let us consider a 2-state $\operatorname{VARMA}(p, q)$ model with Markov regime as in (1). We propose two methods of computation for the spectral density matrix $F_{\mathbf{x}}(\omega)$. First, from Formula (3) we can evaluate the series

$$
\sum_{k=-\infty}^{+\infty} P^{*|k|} e^{-i \omega k}=I_{2 K(p+q)}+2 \mathcal{R} e\left(\sum_{k=1}^{+\infty} P^{* k} e^{-i \omega k}\right)
$$

For every $k \geq 1$, we have by induction

$$
P^{* k}=\left(\begin{array}{ll}
p_{11} \Phi(1) & p_{21} \Phi(1) \\
p_{12} \Phi(2) & p_{22} \Phi(2)
\end{array}\right)^{k}=\left(\begin{array}{cc}
\Phi(1) S_{k} & p_{21} \Phi(1) R_{k} \\
p_{12} \Phi(2) U_{k} & \Phi(2) T_{k}
\end{array}\right)
$$

where

$$
\begin{gathered}
S_{k+1}=p_{11} S_{k} \Phi(1)+p_{12} p_{21} R_{k} \Phi(2) \\
R_{k+1}=S_{k} \Phi(1)+p_{22} R_{k} \Phi(2) \\
U_{k+1}=p_{11} U_{k} \Phi(1)+T_{k} \Phi(2) \\
T_{k+1}=p_{12} p_{21} U_{k} \Phi(1)+p_{22} T_{k} \Phi(2)
\end{gathered}
$$

and $S_{1}=p_{11} I_{K(p+q)}, R_{1}=U_{1}=I_{K(p+q)}$ and $T_{1}=p_{22} I_{K(p+q)}$.

Setting

$$
\begin{array}{rlrl}
x(\omega) & =\sum_{k=1}^{+\infty} R_{k} e^{-i \omega k} & y(\omega) & =\sum_{k=1}^{+\infty} S_{k} e^{-i \omega k} \\
z(\omega) & =\sum_{k=1}^{+\infty} T_{k} e^{-i \omega k} & u(\omega) & =\sum_{k=1}^{+\infty} U_{k} e^{-i \omega k}
\end{array}
$$

we get

$$
F_{\mathbf{x}}(\omega)=\left(g^{\prime} \otimes f^{\prime}\right)\left(\begin{array}{cc}
I_{K(p+q)}+2 \Phi(1) \mathcal{R} e[x(\omega)] & 2 p_{21} \Phi(1) \mathcal{R} e[y(\omega)] \\
2 p_{12} \Phi(2) \mathcal{R} e[u(\omega)] & I_{K(p+q)}+2 \Phi(2) \mathcal{R} e[z(\omega)]
\end{array}\right) \mathcal{W}(0) f
$$

where $g=\left(\begin{array}{ll}1 & 1\end{array}\right)^{\prime}$ and $f^{\prime}=\left(\begin{array}{lll}I_{k} & 0 & \ldots 0\end{array}\right) \in \mathcal{M}(K, K(p+q) ; \mathbb{R})$. 
From (10) we have

$$
\begin{aligned}
\sum_{k=1}^{+\infty} S_{k+1} e^{-i \omega k} & =p_{11} \sum_{k=1}^{+\infty} S_{k} e^{-i \omega k} \Phi(1)+p_{12} p_{21} \sum_{k=1}^{+\infty} R_{k} e^{-i \omega k} \Phi(2) \\
& =p_{11} y(\omega) \Phi(1)+p_{12} p_{21} x(\omega) \Phi(2) .
\end{aligned}
$$

Setting $h=k+1$, we obtain

$$
\begin{aligned}
\sum_{k=1}^{+\infty} S_{k+1} e^{-i \omega k} & =e^{i \omega} \sum_{h=2}^{+\infty} S_{h} e^{-i \omega h}=e^{i \omega}\left(-S_{1} e^{-i \omega}+\sum_{h=1}^{+\infty} S_{h} e^{-i \omega h}\right) \\
& =e^{i \omega}\left(-p_{11} I_{K(p+q)} e^{-i \omega}+y(\omega)\right)=-p_{11} I_{K(p+q)}+e^{i \omega} y(\omega)
\end{aligned}
$$

Equating the last two formulae and solving for $y(\omega)$ yield

$$
y(\omega)=\left(p_{11} I_{K(p+q)}+p_{12} p_{21} x(\omega) \Phi(2)\right)\left(I_{K(p+q)} e^{i \omega}-p_{11} \Phi(1)\right)^{-1} .
$$

Here we have

$$
I_{K(p+q)} e^{i \omega}-p_{11} \Phi(1)=\left(\begin{array}{cc}
I_{K p} e^{i \omega}-p_{11} A(1) & -p_{11} B(1) \\
0 & I_{K q} e^{i \omega}-p_{11} \mathbf{J}
\end{array}\right)
$$

hence the Woodbury formula gives

$$
\left(I_{K(p+q)} e^{i \omega}-p_{11} \Phi(1)\right)^{-1}=\left(\begin{array}{cc}
\left(I_{K p} e^{i \omega}-p_{11} A(1)\right)^{-1} & Q \\
0 & \left(I_{K q} e^{i \omega}-p_{11} \mathbf{J}\right)^{-1}
\end{array}\right)
$$

where

$$
Q=p_{11}\left(I_{K p} e^{i \omega}-p_{11} A(1)\right)^{-1} B(1)\left(I_{K q} e^{i \omega}-p_{11} \mathbf{J}\right)^{-1} .
$$

Reasoning as above, from (11), (12) and (13) we get respectively

$$
\begin{gathered}
x(\omega)=\left(I_{K(p+q)}+y(\omega) \Phi(1)\right)\left(I_{K(p+q)} e^{i \omega}-p_{22} \Phi(2)\right)^{-1} \\
u(\omega)=\left(I_{K(p+q)}+z(\omega) \Phi(2)\right)\left(I_{K(p+q)} e^{i \omega}-p_{11} \Phi(1)\right)^{-1} \\
z(\omega)=\left(p_{22} I_{K(p+q)}+p_{12} p_{21} u(\omega) \Phi(1)\right)\left(I_{K(p+q)} e^{i \omega}-p_{22} \Phi(2)\right)^{-1} .
\end{gathered}
$$

From (14) and (15) we obtain

$$
\begin{aligned}
x(\omega)= & {\left[I_{K(p+q)}+p_{11}\left(I_{K(p+q)} e^{i \omega}-p_{11} \Phi(1)\right)^{-1} \Phi(1)\right] } \\
& \times\left[I_{K(p+q)} e^{i \omega}-p_{22} \Phi(2)-p_{12} p_{21} \Phi(2)\left(I_{K(p+q)} e^{i \omega}-p_{11} \Phi(1)\right)^{-1} \Phi(1)\right]^{-1} .
\end{aligned}
$$

Substituting this formula into (14) gives $y(\omega)$. From (16) and (17) we get

$$
\begin{aligned}
u(\omega)= & {\left[I_{K(p+q)}+p_{22}\left(I_{K(p+q)} e^{i \omega}-p_{22} \Phi(2)\right)^{-1} \Phi(2)\right] } \\
& \times\left[I_{K(p+q)} e^{i \omega}-p_{11} \Phi(1)-p_{12} p_{21} \Phi(1)\left(I_{K(p+q)} e^{i \omega}-p_{22} \Phi(2)\right)^{-1} \Phi(2)\right]^{-1} .
\end{aligned}
$$

Substituting this formula into (17) gives $z(\omega)$.

As an alternative method for computing $F_{\mathbf{x}}(\omega)$, let us consider $X(\omega)=\left(I_{2 K(p+q)} e^{i \omega}-P^{*}\right)^{-1} e^{i \omega}$ from Theorem 2 . Then we have

$$
I_{2 K(p+q)} e^{i \omega}-P^{*}=\left(\begin{array}{cc}
A_{11} & A_{12} \\
A_{21} & A_{22}
\end{array}\right)
$$

where

$$
\begin{array}{lrl}
A_{11}=I_{K(p+q)} e^{i \omega}-p_{11} \Phi(1) & A_{12}=-p_{21} \Phi(1) \\
A_{21}=-p_{12} \Phi(2) & A_{22}=I_{K(p+q)} e^{i \omega}-p_{22} \Phi(2) .
\end{array}
$$

By Woodbury formula we obtain

$$
\left(I_{2 K(p+q)} e^{i \omega}-P^{*}\right)^{-1}=\left(\begin{array}{cc}
A_{11}^{-1}+A_{11}^{-1} A_{12} \Omega_{22} A_{21} A_{11}^{-1} & -A_{11}^{-1} A_{12} \Omega_{22} \\
-\Omega_{22} A_{21} A_{11}^{-1} & \Omega_{22}
\end{array}\right)
$$

where

$$
\Omega_{22}=\left[I_{K(p+q)} e^{i \omega}-p_{22} \Phi(2)-p_{12} p_{21} \Phi(2)\left(I_{K(p+q)} e^{i \omega}-p_{11} \Phi(1)\right)^{-1} \Phi(1)\right]^{-1}
$$


Taking $\Omega=I_{K}$, we have

$$
V=\operatorname{vec} \mathcal{W}(0)=\left(I_{2 K^{2}(p+q)^{2}}-P\right)^{-1} \widetilde{S}
$$

where

$$
\widetilde{S}=\left(\begin{array}{l}
\pi_{1} \operatorname{vec}\left(\Sigma \Sigma^{\prime}\right) \\
\pi_{2} \operatorname{vec}\left(\Sigma \Sigma^{\prime}\right)
\end{array}\right)
$$

and

$$
\Sigma=\left(\begin{array}{llllllll}
\sigma_{\epsilon}^{2} I_{K} & 0 & \cdots & 0 & \sigma_{\epsilon}^{2} I_{K} & 0 & \cdots & 0
\end{array}\right)^{\prime} \in \mathcal{M}(K(p+q), K ; \mathbb{R}) .
$$

Here we set

$$
I_{2 K^{2}(p+q)^{2}}-P=\left(\begin{array}{ll}
B_{11} & B_{12} \\
B_{21} & B_{22}
\end{array}\right)
$$

where

$$
\begin{array}{ll}
B_{11}=I_{K^{2}(p+q)^{2}}-p_{11} \Phi(1) \otimes \Phi(1) & B_{12}=-p_{21} \Phi(1) \otimes \Phi(1) \\
B_{21}=-p_{12} \Phi(2) \otimes \Phi(2) & B_{22}=I_{K^{2}(p+q)^{2}}-p_{22} \Phi(2) \otimes \Phi(2) .
\end{array}
$$

By Woodbury formula we obtain

$$
\left(I_{2 K^{2}(p+q)^{2}}-P\right)^{-1}=\left(\begin{array}{cc}
B_{11}^{-1}+B_{11}^{-1} B_{12} \Lambda_{22} B_{21} B_{11}^{-1} & -B_{11}^{-1} B_{12} \Lambda_{22} \\
-\Lambda_{22} B_{21} B_{11}^{-1} & \Lambda_{22}
\end{array}\right)
$$

where $\Lambda_{22}=\left(B_{22}-B_{21} B_{11}^{-1} B_{12}\right)^{-1}$.

\section{Examples and numerical applications}

(3.1). We start with some 2-state univariate MS(2)-AR(1) models, considered in Pataracchia (2008), Section 3, pp.11-13, and report her interesting observations developed there to make the reading self-contained. In Figure 1 the solid line represents the spectral density functions of $\operatorname{MS}(2)-\operatorname{AR}(1)$ models and the dashed lines denote the spectra of the two underlying states. The variance of the error term is state invariant and we set $\sigma_{\epsilon}^{2}=1$. For $p_{11}=p_{22}=0.5, a(1)=0.8$ and $a(2)=0.2$, we have $\rho(P)=0.34$ and $\rho\left(P^{*}\right)=0.5$. The spectra are shown in Figure 1 (a). For $p_{11}=0.3, p_{22}=0.8, a(1)=0.8$ and $a(2)=0.2$, we get $\rho(P) \sim 0.21$ and $\rho\left(P^{*}\right) \sim 0.35$. The spectra are shown in Figure 1(b). Such figures correspond to two MS(2)-AR(1) models with identical coefficients but different transition probability matrices. They show that the spectral density of a regime switching model presents itself as a sort of weighted average of the spectra of the underlying models. As the less volatile model becomes more likely (for instance in Figure 1(b)), the peak of the spectral density function decreases. In Figure 1(c) we show the spectral density of an $\operatorname{MS}(2)-\operatorname{AR}(1)$ with $p_{11}=p_{22}=0.5, a(1)=-0.8$ and $a(2)=-0.2$. When both $a(1)$ and $a(2)$ are positive (resp. negative), the spectral shape is similar to a typical spectral representation of a stable AR(1) with positive (resp. negative) coefficients. When the sign of the coefficients is opposite, the prevailing shape depends on which model dominates the other, in terms of both the underlying unconditional probabilities and the absolute values of its coefficients. See Figure $1(\mathrm{~d})$, where $p_{11}=p_{22}=0.5, a(1)=0.8$ and $a(2)=-0.2$.

(3.2). Let us consider a 2 -state univariate $\operatorname{MS}(2)-\operatorname{AR}(1)$ model with state invariant standard error $\sigma_{\epsilon}=0.5$ $(=\sigma(1)=\sigma(2)), a(1)=0.25, a(2)=-1.9, p_{11}=0.1$ and $p_{22}=0.2=1-p_{21}$. See Francq and Zakoïan (2001), Section 6, pp.357-362. In this case, we have $\rho(P) \sim 0.90$ and $\rho\left(P^{*}\right) \sim 0.58$. The stationary probability $\pi_{1} \sim 0.47$ and $\pi_{2} \sim 0.53$ are close to 0.5. The spectra are shown in Figure 2. The global stationarity is met but not the local one. The simulations results, obtained in the quoted paper (see Tables 3 and 4, pp.362-363), are reported in Table 1 below. The first column contains the true values of the parameters. In the second column there are the parameter estimates which are obtained without stationarity constraints (wsc). The third (resp. fourth) column shows estimation under the global (resp. local) stationarity constraints, in short gsc (resp. lsc).

The estimations obtained without imposing any stationarity constraint are slightly better than those obtained under the global or local stationarity constraints. This is confirmed by the closest position of the spectral density to the true one.

(3.3). Let us consider the following univariate $\operatorname{MS}(2)-\operatorname{AR}(2)$ model

$$
x_{t}=\left\{\begin{array}{lcc}
\eta_{t} & \text { if } & \xi_{t}=1 \\
a x_{t-2}+\eta_{t} & \text { if } & \xi_{t}=2
\end{array}\right.
$$




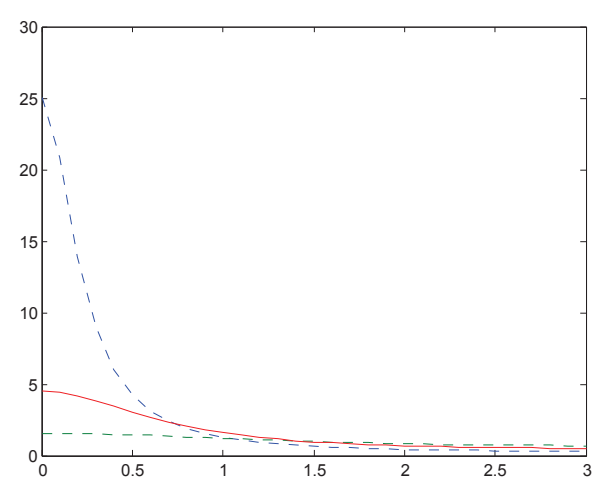

(a)

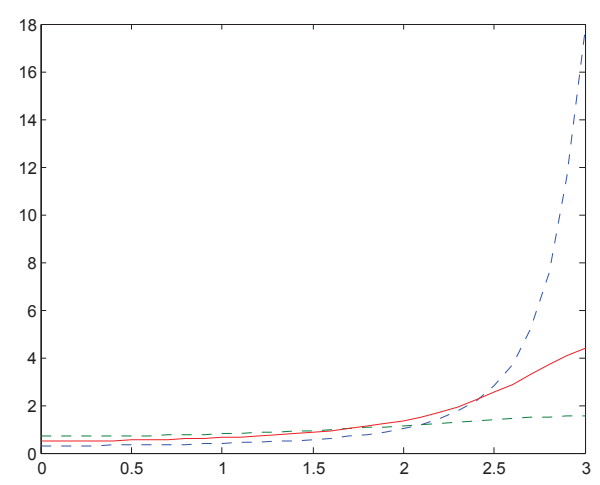

(c)

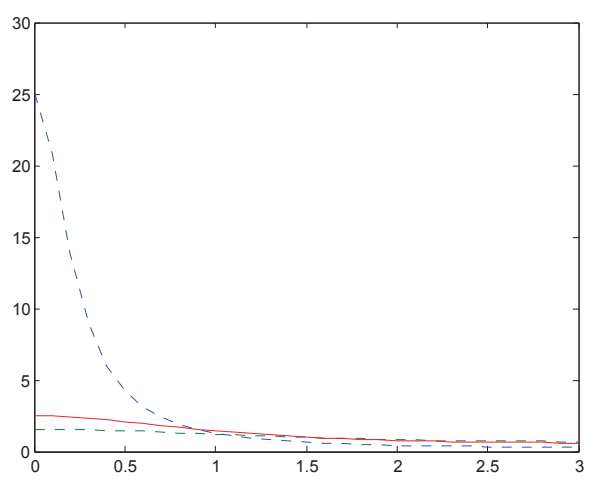

(b)

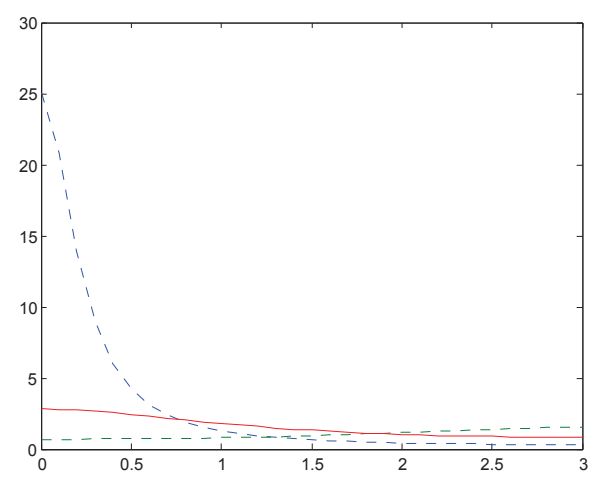

(d)

Figure 1. Solid line: spectral density of an univariate MS(2)-AR(1); Dashed line: spectral densities of the two underlying linear models. Parameters are set as in $\S 3.1$.

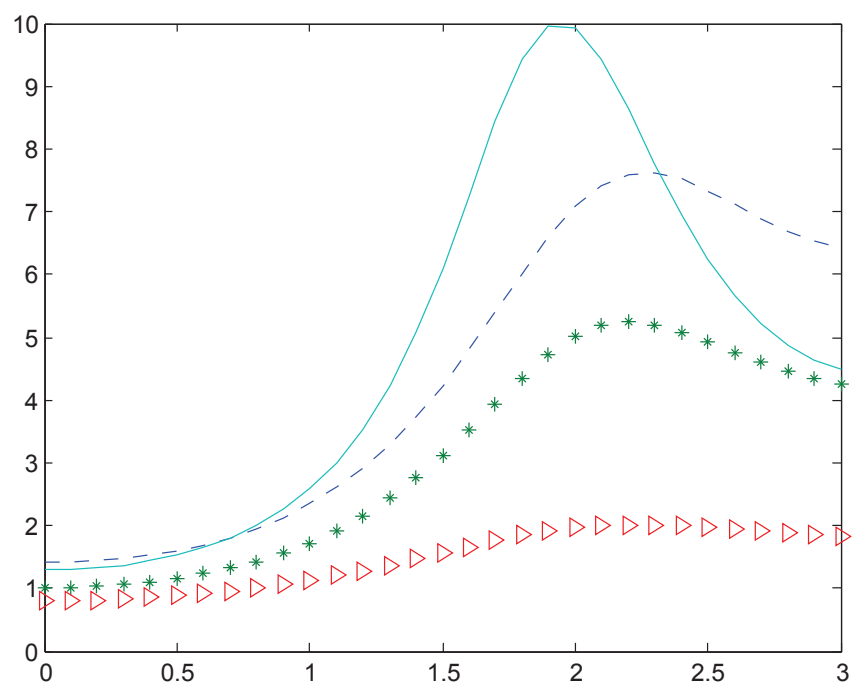

Figure 2. Spectral density functions of the univariate $\operatorname{MS}(2)-\operatorname{AR}(2)$ as in Francq and Zakoïan (2001). The lines are spectral densities evaluated in the true parameters (solid line), in the estimated parameters without stationarity constraints (wsc - dashed line),global stationarity constraints (gsc - starred line) and local stationarity constraints (lsc - triangle). It refers to Example $\S 3.2$. 
Table 1. Parameters values of the univariate $\mathrm{MS}(2)-\mathrm{AR}(2)$ as in Francq and Zakoïan (2001). The true parameter of the model are in the first column. Estimated parameters obtained in estimation without stationarity constraints (wsc), under global stationarity constraints (gsc) and local stationarity constraints (lsc) are reported also

\begin{tabular}{|l|c|c||c|}
\hline true & wsc & gsc & lsc \\
\hline$p_{11}=0.1$ & 0.190 & 0.191 & 0.140 \\
$p_{21}=0.8$ & 0.738 & 0.754 & 0.691 \\
$a(1)=0.25$ & 0.208 & 0.206 & 0.211 \\
$a(2)=-1.9$ & -1.718 & -1.695 & -0.933 \\
$\sigma(1)=0.5$ & 0.506 & 0.508 & 0.491 \\
$\sigma(2)=0.5$ & 0.806 & 0.820 & 1.250 \\
\hline
\end{tabular}

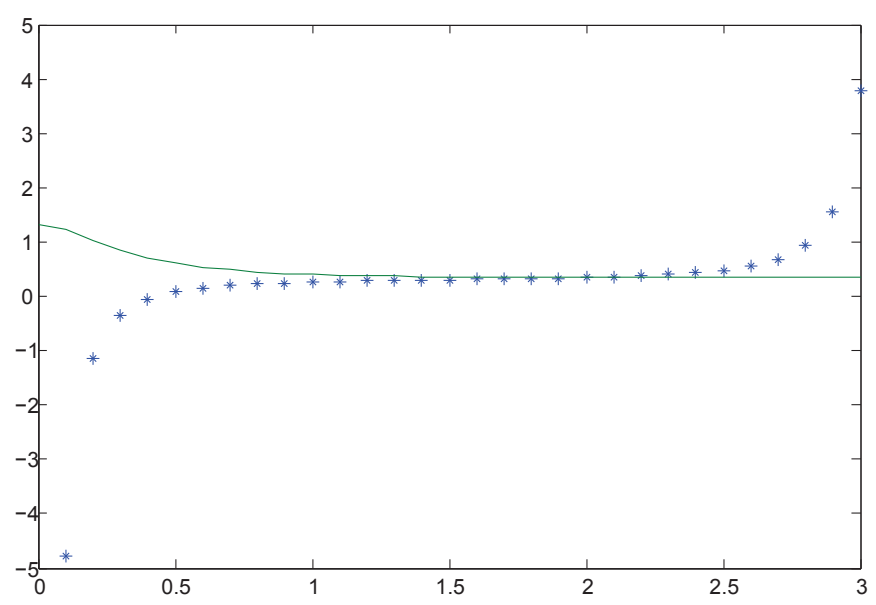

Figure 3. Spectral densities of univariate $\operatorname{MS}(2)-\operatorname{AR}(2)$ as in Example $§ 3.3$. Solid line is the spectral density of the model not satisfying local stationarity; starred line is the spectra corresponding to the model both local and global stationary.

where $\eta_{t} \sim \operatorname{IID}(0,1)$ and $a$ is a nonzero real constant. See Francq and Zakoïan (2001), Example 4, p.352. Then we have

$$
\Phi(1)=A(1)=\left(\begin{array}{ll}
0 & 0 \\
1 & 0
\end{array}\right) \quad \Phi(2)=A(2)=\left(\begin{array}{ll}
0 & a \\
1 & 0
\end{array}\right) .
$$

The spectral radii of the matrices $P$ and $P^{*}$ are given by

$$
\rho(P)=|a| \sqrt{p_{22}^{2}+p_{12} p_{21}} \quad \rho\left(P^{*}\right)=\sqrt{|a|} \sqrt{p_{22}^{2}+p_{12} p_{21}} .
$$

We know that $\rho(P)<1$ implies $\rho\left(P^{*}\right)<1$. The converse is not true in general. For example, if $p_{11}=0.1, p_{22}=0.2$ and $a=1.2$, then $\rho(P) \sim 1.05$ and $\rho\left(P^{*}\right) \sim 0.95$. However, if $|a|<1$, then $\rho(P)$ and $\rho\left(P^{*}\right)$ are both less than one. Spectra are shown in Figure 3. Both models are global stationarity. However, the former does not satisfy local stationary, while the latter does. Differences between the two spectra appear at the extremes of the cycle.

(3.4). Let us consider the following univariate $\mathrm{MS}(2)-\mathrm{AR}(2)$ model

$$
x_{t}=a_{1}\left(\xi_{t}\right) x_{t-1}+a_{2}\left(\xi_{t}\right) x_{t-2}+\sigma(\xi) \eta_{t}
$$

where $\eta_{t} \sim \operatorname{IID}(0,1)$. For $a_{1}(1)=1$, and also for greater values, $a_{2}(1)=0.8, a_{1}(2)=-2, a_{2}(2)=0.5, \sigma(1)=1$ and $\sigma(2)=0.25$, the underlying processes are non stationary. However, the resulting Markov switching process is global stationary. See Krishnamurthy and Ryden (1998), Example 5. In fact, we have

$$
\Phi(1)=A(1)=\left(\begin{array}{cc}
1 & 0.8 \\
1 & 0
\end{array}\right) \quad \Phi(2)=A(2)=\left(\begin{array}{cc}
-2 & 0.5 \\
1 & 0
\end{array}\right)
$$

hence $\rho(\Phi(1)) \sim 1.52$ and $\rho(\Phi(2)) \sim 2.22$. For $p_{11}=p_{22}=0.01$, we get $\rho(P) \sim 0.69$ and $\rho\left(P^{*}\right) \sim 0.79$. If we change $a_{1}(1)$ into -0.03 , the resulting process is not stationary. In this case, we have $\rho(P) \sim 1.02$ and $\rho\left(P^{*}\right) \sim 0.98$. Spectra are shown in Figure 4. The former process is global stationary and its spectral density appears to be quite flat; on the contrary, the latter process which does not satisfy global stationary has a inverted-U shape spectral density function.

(3.5). For a general Markov switching autoregressive model of order $p \geq 2$, local stationarity does not imply global stationarity. The following univariate MS(2)-AR(2) model was considered by Francq and Zakoïan (2001), Example 


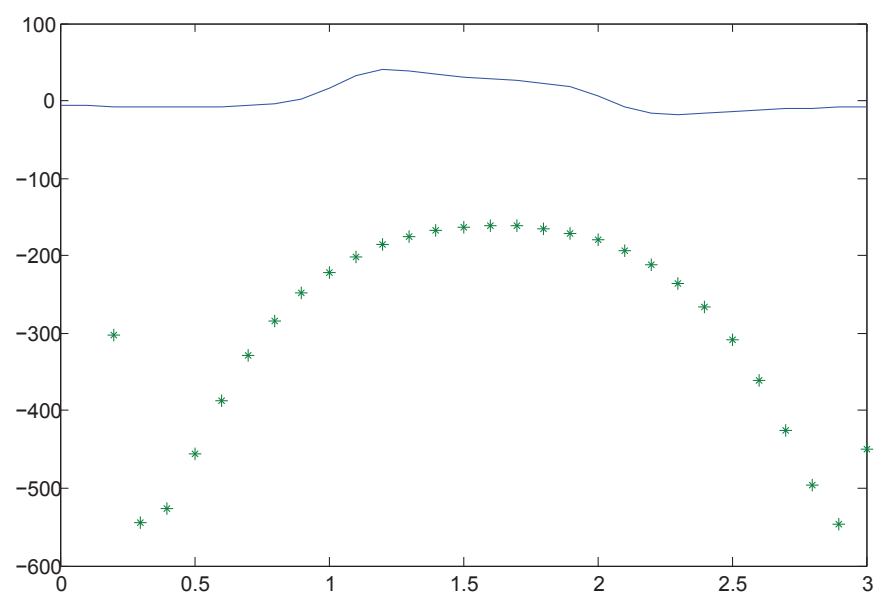

Figure 4. Spectral density of univariate $\operatorname{MS}(2)-\operatorname{AR}(2)$ : the process is global stationary (solid line) and no global stationary (starred line).

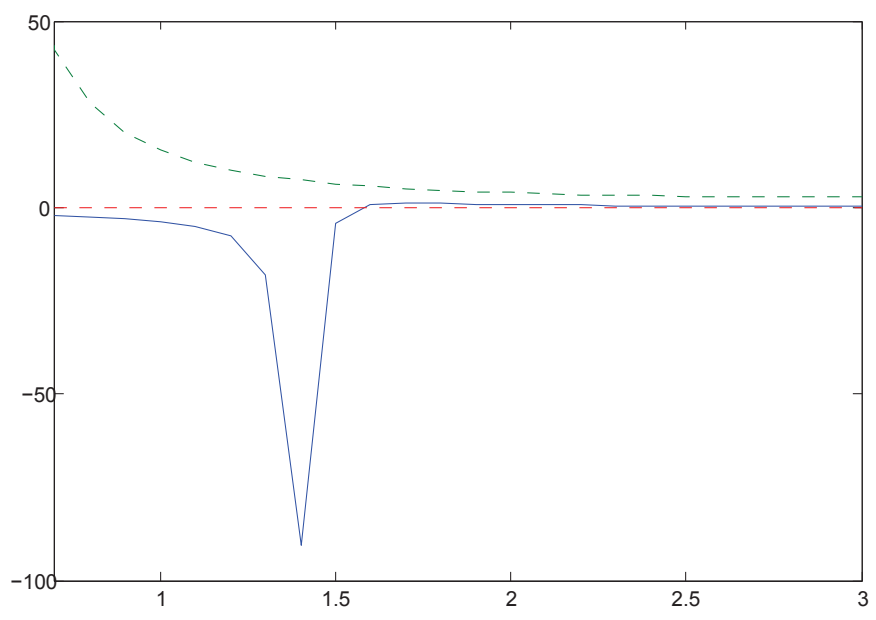

Figure 5. Spectral density of univariate $\mathrm{MS}(2)-\mathrm{AR}(2)$ which is explosive (solid line). The dashed lines are the two underlying linear models. Parameters as in $§ 3.5$.

5, p.353:

$$
x_{t}=\left\{\begin{array}{lll}
a_{1}(1) x_{t-1}+a_{2}(1) x_{t-2}+\eta_{t} & \text { if } & \xi_{t}=1 \\
a_{1}(2) x_{t-1}+\eta_{t} & \text { if } & \xi_{t}=2
\end{array}\right.
$$

where $\eta_{t} \sim \operatorname{IID}(0,1)$. Then we have

$$
\Phi(1)=A(1)=\left(\begin{array}{cc}
a_{1}(1) & a_{2}(1) \\
1 & 0
\end{array}\right) \quad \Phi(2)=A(2)=\left(\begin{array}{cc}
a_{1}(2) & 0 \\
1 & 0
\end{array}\right) .
$$

For $p_{11}=0.2, p_{22}=0.1, a_{1}(1)=1.8, a_{2}(1)=-0.9$ and $a_{1}(2)=-0.2$, both regimes are stationary (in fact, $\rho(\Phi(1)) \sim 0.95$ and $\rho(\Phi(2))=0.2)$ but the resulting process is explosive (the explosion is exponential). See Figure 5 for the spectrum, which shows quite a striking peak. Because the probability of staying in the same regime is small, the local stationarity does not compensate the explosive nature of the regime changes as remarked in the quoted paper at p.353. In this case we have $\rho(P) \sim 1.33$ and $\rho\left(P^{*}\right) \sim 0.96$. The spectral density could be then first step to immediatly evaluate the behavior of such an explosive process.

(3.6). Let us consider the following univariate MS(3)-ARMA $(1,2)$ model

$$
x_{t}=\left\{\begin{array}{lcc}
1.5 x_{t-1}+\eta_{t}+0.5 \eta_{t-1}-1.35 \eta_{t-2} & \text { if } & \xi_{t}=1 \\
0.9 x_{t-1}+\eta_{t}-1.8 \eta_{t-2} & \text { if } & \xi_{t}=2 \\
\eta_{t}+0.9 \eta_{t-1} & \text { if } & \xi_{t}=3
\end{array}\right.
$$




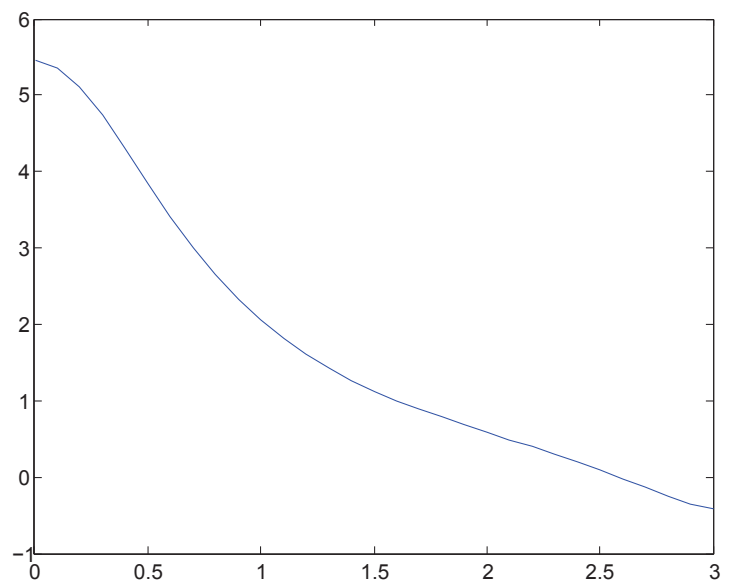

Figure 6. Spectral density of a univariate MS(3)-ARMA $(1,2)$ model. Parameters as in $§ 3.6$.

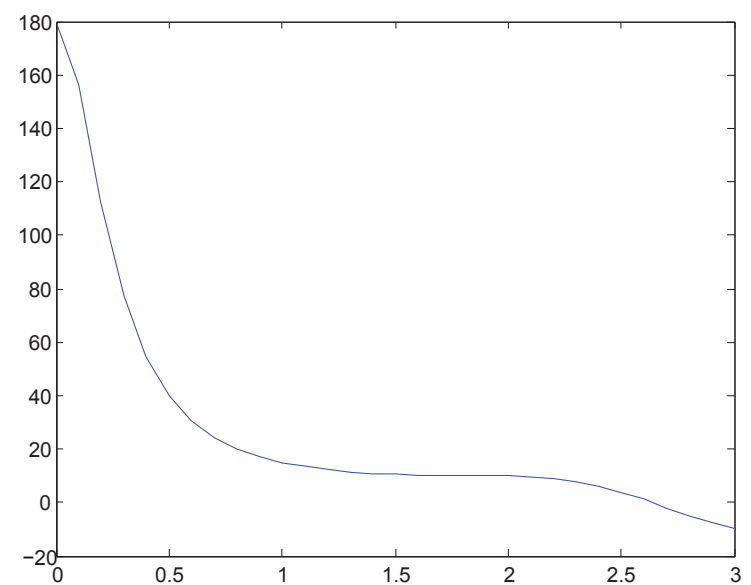

Figure 7. Spectral density of a univariate $\operatorname{MS}(3)-\operatorname{ARMA}(2,1)$ model.

where $\eta_{t} \sim \operatorname{IID}(0,1)$. See Francq and Zakoïan (2001), Example 6, pp.354-355. Then we have

$$
\Phi(1)=\left(\begin{array}{ccc}
1.5 & 0.5 & -1.35 \\
0 & 0 & 0 \\
0 & 1 & 0
\end{array}\right) \quad \Phi(2)=\left(\begin{array}{ccc}
0.9 & 0 & -1.8 \\
0 & 0 & 0 \\
0 & 1 & 0
\end{array}\right)
$$

and

$$
\Phi(3)=\left(\begin{array}{ccc}
0 & 0.9 & 0 \\
0 & 0 & 0 \\
0 & 1 & 0
\end{array}\right)
$$

hence $\rho(\Phi(1))=1.5, \rho(\Phi(2))=0.9$ and $\rho(\Phi(3))=0$. For $p_{11}=p_{22}=p_{32}=0$ and $p_{13} \neq 0$, we have $\rho(P) \sim 1.16$ and $\rho\left(P^{*}\right) \sim 1.16$. This model has a second-order stationary solution. Thus $\rho(P)<1$ is only a sufficient secondorder stationarity condition in the general case. If $p_{12}=0.8, p_{23}=0.8$ and $p_{31}=0.8$, then $\rho(P)=0.54$ and $\rho\left(P^{*}\right)=0.4648$. In this case, the stationary probabilities are $\pi_{1}=0.3509, \pi_{2}=0.2807$ and $\pi_{3}=0.3684$. The spectral density for the last model are shown in Figure 6.

Let us compare the above spectrum with a similarly constructed spectrum for an MS(3)-ARMA $(2,1)$ model (see Figure 7). When the autoregressive prevails as in this case, the spectral density assume the common shape of autoregressive process. While in Figure 6 the shape was mostly similar to the spectral density of a moving average process.

\section{REFERENCES}

[1] Cavicchioli, M. 2013. Spectral density of Markov switching VARMA. Economics Letters, Vol. 121, 218-220. 
[2] Costa, O.L.V., Fragoso, M.D., Marques, R.P. 2005. Discrete-Time Markov Jump Linear Systems. in: Probability and Its Applications (Gani, J., Heyde, C.C., Jagers, P., and Kurtz, T.G., eds.) Springer-Verlag, London.

[3] Francq, C., Zakoïan, J.M. 2001. Stationarity of multivariate Markov switching ARMA models. Journal of Econometrics 102, 339-364.

[4] Hamilton, J.D. 1989. A new approach to the economic analysis of nonstationary time series and the business cycle. Econometrica 57, 357-384.

[5] Hamilton, J.D. 1990. Analysis of time series subject to changes in regime. Journal of Econometrics 45, 39-70.

[6] Horn, R.A., Johnson, C.R. 1985. Matrix Analysis. Cambridge Univ. Press, Cambridge-London-New York.

[7] Horn, R.A., Johnson, C.R. 1994. Topics in Matrix Analysis. Cambridge Univ. Press, Cambridge-London-New York.

[8] Krishnamurthy, V., Ryden, T. 1998. Consistent estimation of linear and non-linear autoregressive models with Markov regime. Journal of Time Series Analysis 19 (3), 291-307.

[9] Pataracchia, B. 2008. The spectral representation of Markov switching ARMA models. In: $5^{\text {th }}$ Eurostat Colloquium on Modern Tools for Business Cycle Analysis (Luxembourg, 29 September-1 October 2008), jointly organised by EUROSTAT and European Univ. Institute, Florence, Italy.

[10] Pataracchia, B. 2011. The spectral representation of Markov switching ARMA models. Economics Letters 112, 11-15.

[11] Stelzer, R. 2009. On Markov-switching ARMA processes-stationarity, existence of moments and geometric ergodicity. Econometric Theory 25 (1), 43-62.

[12] Xie, Y., Yu, J., Ranneby, B. 2008. A general autoregressive model with Markov switching: estimation and consistency. Mathematical Methods of Statistics 17 (3), 228-240. 